\title{
Early Clinical Results Using Normothermic Machine Liver Preservation
}

\author{
Vinzent N. Spetzler • Nicolas Goldaracena • \\ Nazia Selzner • Markus Selzner
}

Published online: 11 January 2015

(C) Springer International Publishing AG 2015

\begin{abstract}
The ongoing shortage of donor organs for liver transplantation results in high mortality rates for those on the waiting list. This has triggered considerable interest in the use of marginal liver grafts to increase the donor pool. Unfortunately, marginal grafts poorly tolerate preservation by cold storage (CS), resulting in an increased risk of liver injury and poor graft function after transplantation. Better preservation techniques are needed to fully utilize marginal grafts and to expand the number of organ donors. Animal studies have demonstrated normothermic ex vivo liver perfusion to be superior to conventional CS. Recently, the first clinical trial of normothermic ex vivo liver perfusion was completed with promising results. Similarly, in situ recirculation after cardiac arrest as the initial preservation method before organ procurement has proven to be a valuable technique to make more liver grafts available for transplantation. Though technically more complex and economically more expensive, normothermic liver perfusion is a promising and
\end{abstract}

This article is part of the Topical Collection on Machine Preservation of the Liver

V. N. Spetzler $\cdot$ N. Goldaracena $\cdot$ M. Selzner

Department of Surgery, Multi Organ Transplant Program, Toronto

General Hospital, Toronto, ON, Canada

V. N. Spetzler

e-mail: vinzent.spetzler@gmail.com

N. Goldaracena

e-mail: n.goldaracena@yahoo.com

N. Selzner

Department Medicine, Multi Organ Transplant Program, Toronto

General Hospital, Toronto, ON, Canada

e-mail: nazia.selzner@uhn.ca

M. Selzner $(\square)$

Toronto General Hospital, NCSB 11C-1244, 585 University Avenue,

Toronto, ON M5G2N2, Canada

e-mail: markus.selzner@uhn.ca innovative technique that is able to positively impact current transplant practice and outcomes.

Keywords Liver transplantation $\cdot$ Ex vivo liver perfusion . In situ liver perfusion $\cdot$ Normothermic perfusion .

Normothermic recirculation - Ischemic type biliary strictures . preservation/reperfusion injury

\section{Introduction}

Before the invention of cold static storage (CS) solutions in the early 1970s [1], organ perfusion was the gold standard for liver graft preservation [2]. Since then, however, CS has become the preferred method of preservation because it is easy to apply, relatively low cost, and provides acceptable results after transplantation with good-quality organs. CS is based on the concept of hypothermia and its underlying reduction of metabolism and oxygen demand. These factors prolong organ viability by slowing down progression of ischemic injury. However, because cellular metabolism is not completely stopped at $4{ }^{\circ} \mathrm{C}$, cold-stored grafts eventually develop an energy debt with depletion of adenosine triphosphate (ATP) stores [3]. ATP depletion results in dysfunction of the $\mathrm{Na}+/ \mathrm{K}+$ cell membrane pumps, accumulation of toxic products derived from anaerobic metabolism, mitochondrial injury, and cell swelling [4-6]. Subsequently, at the time of normothermic graft reperfusion, exceeding oxygen supply to dysfunctional mitochondria results in the generation of reactive oxygen species, increased cellular damage, and the activation of pro-inflammatory pathways [7-10]. Depending on the initial quality of the organ graft and the duration of cold ischemic injury, the damage of this ischemia-reperfusion cascade ranges from minor cellular dysfunction, to ischemic cholangiopathy, to primary graft failure [11-13]. 
The severe organ shortage over the last two decades has triggered an interest in increasing the donor pool by expanding donor criteria. As a consequence, marginal grafts are more frequently accepted for transplantation $[14,15]$. These extended criteria organs include grafts donated after cardio-circulatory death (DCD), steatotic livers, livers from elderly donors, and grafts with prolonged CS time. Unfortunately, extended criteria grafts are more susceptible to ischemic injury during CS and are more likely to develop graft dysfunction and biliary complications after transplantation [16, 17]. In addition, CS does not allow organ assessment, with the acceptance or decline of liver grafts based on personal opinion rather than objective data. Over the last two decades, cardiopulmonary machines and oxygenators have significantly advanced, providing better technology for organ perfusion. The shortcomings of CS and advances in perfusion technology have resulted in increased interest in normothermic perfused organ preservation as an alternative to CS.

\section{Principles of Normothermic Liver Perfusion}

The main goal of normothermic machine perfusion (NMP) is to optimize graft preservation by mimicking physiological conditions. The perfused organ is supplied with nutrients and oxygen to maintain metabolic hemostasis. At the same time, toxic products from the cellular milieu are continuously eliminated. Under these conditions, ATP and glycogen reserves can be maintained or actively restored [18]. As long as the perfusate does not contain pro-inflammatory mediators, such as cytokines, leucocytes or platelets, the cell-mediated injury phase of reperfusion injury can be minimized. Thus, ischemic injury is avoided and the activation of cell death cascades is prevented. This allows both hepatocellular and biliary protection [19•, 20, 21]. In addition, previously injured organs, such as organs exposed to prolonged warm ischemia, have the opportunity to initiate cellular repair regeneration pathways.

Similarly important to the prevention of preservation injury and optimal transplant outcomes is the ability to assess organs during ex vivo NMP. Since the organ is metabolically active, its quality can be evaluated by vascular flow parameters, injury markers (i.e., transaminases), and functional indicators such as bile production and metabolic activity [22, 23]. By assessing graft injury and metabolic function during organ perfusion, transplant physicians and surgeons can accept or decline liver grafts based on data, instead of historical information and macroscopic appearance. Furthermore, the active metabolism during ex vivo NMP also offers the opportunity to apply repair strategies to improve the quality of marginal grafts [18].
Technical Aspects of Normothermic Liver Perfusion

While the first human trial with normothermic ex vivo liver perfusion was just recently initiated, clinical studies with ex vivo NMP have already been performed in other organ systems such as lung [24, 25], heart [26], and kidney [27]. The delay in initiating clinical studies in normothermic liver perfusion is partially due to the organ's complexity and high metabolic demand.

In contrast to other organs, liver perfusion involves two separate inflow vessels (hepatic artery and portal vein) with different pressures and flow requirements. While the hepatic artery requires a high pressure $(50-70 \mathrm{mmHg})$ with a moderate flow $(300-600 \mathrm{~mL} / \mathrm{min})$, the portal venous system requires a high flow $(600-900 \mathrm{~mL} / \mathrm{min})$ with low inflow pressure (3$5 \mathrm{mmHg}$ ). Most studies have used continuous flow as opposed to a pulsatile flow in the hepatic artery, but there are no data available supporting the superiority of either flow setting. However, clinical experiences from left ventricular assist devices demonstrate a decreased technical liability of the continuous flow technique with comparable functional results [28], suggesting similar implications for normothermic liver perfusion. For liver NMP, the venous blood can drain openly into the organ basin [29] from where it is recirculated or it can pass through a closed tubing system toward a reservoir coming from both supra- and infrahepatic vena cava outflow [30, $31]$ or one vena cava cannula [21,32].

Importantly, because of its high metabolism, the liver requires an adequate oxygen supply that cannot be met by a hyperbaric oxygenated perfusate only [33]. While for normothermic lung perfusions sufficient oxygen diffusion is achieved by ventilation, the perfusate in liver NMP requires an additional oxygen carrier. Because of a lack of available alternatives, most research groups have used erythrocytes as the oxygen carrier. While many preclinical animal studies have been conducted with whole blood from the donor animal as perfusate $[21,32,34,35]$, this is not practical for human clinical application because the blood of the organ donor is usually not available. In the clinical setting of a human trial, erythrocytes can be provided by leuko-reduced packed red blood cells (RBCs) supplied by the local blood bank. The RBCs are subsequently mixed with a colloidal perfusion solution to substitute the plasma component of whole blood. Examples for colloidal perfusion solution are fresh frozen plasma [29], albumin-rich Steen solution [19•], or starchbased solutions known as plasma-expander in clinical practice. Additional perfusate components, such as antibacterials, nutrients, anticoagulants, and antioxidants have been used in animal experiments to improve the outcome of liver transplantation with normothermic perfused grafts $[18,36]$.

Ideally, NMP is initiated immediately after organ retrieval in order to avoid any additional cold ischemic injury. This is supported by the findings of Peter Friend's group, which 
demonstrated that CS prior to normothermic liver perfusion increased liver injury with deterioration of metabolic function $[20,37]$. In order to avoid the need for CS in clinical practice, a transportable perfusion machine is required to start ex vivo NMP directly in the donor center. It's important to recognize, however, that liver perfusion during organ transportation adds technical complexity to the perfusion process. Perfusion safety during organ transportation could be problematic because any disruption of warm perfusion might lead to perfusion failure and graft loss. Alternatively, liver grafts could be cold stored for the transport to the transplant center with a delayed start of the normothermic perfusion at the transplant center site. CS followed by ex vivo NMP will likely require a modified perfusion solution to compensate for the inflammatory stimulus of the CS period.

\section{Ex Vivo Perfusion}

\section{Preclinical Studies}

Numerous studies have been performed over the last two decades investigating ex vivo NMP with ex vivo blood reperfusion as a model of liver transplantation. The results of preclinical studies provide a solid foundation and direction for early clinical studies, with particular focus on the positive results NMP demonstrates in terms of survival rates and the degree of graft injury, based on a variety of parameters, including timing and perfusion temperature.

\section{Clinical Experience with Normothermic Ex Vivo Perfusion}

Table 1 provides a summary of normothermic liver perfusion in clinical trials.

The results of a first phase I trial conducted at Kings College Hospital have recently been presented at the 2014 annual meeting of the International Liver Transplantation Society in London, UK [38••]. The goal of the study was to demonstrate safety and feasibility of normothermic ex vivo liver perfusion using a non-randomized prospective study design.

A total of 20 adult human livers were preserved at physiological temperatures in the transportable metra ${ }^{\mathrm{TM}}$ perfusion machine (OrganOx, Oxford, UK). Of these 20 grafts, eight livers were considered as marginal grafts (four DCD livers, two livers with increased hepatic injury markers, one liver from a $>75$-year-old donor, and one steatotic liver). The period of CS in University of Wisconsin solution consisted only of the time needed for the back-table preparation and the time of organ implantation during the recipient operation. Organs were brought to the transplant center by road-bound transportation.

The $m e t r a^{\mathrm{TM}}$ perfusion device uses a pressure-controlled, continuous perfusion technique via the hepatic artery and 
portal vein. The venous outflow drains into a soft-shell reservoir through the infrahepatic cava only, while the suprahepatic caval ostium is closed by a continuous suture. The perfusate consisted of $500 \mathrm{~mL}$ of a colloidal solution and 3 units of RBCs. Heparin, cefuroxime, calcium gluconate, and sodium bicarbonate were added during priming of the machine, while insulin, taurocholic acid, heparin, and nutrients were continuously infused during the graft perfusion. Prostacyclin was used as vasodilator. The perfusion set-up allowed stable preservation for up to $18 \mathrm{~h}$ with steady arterial and portal venous flows, bile production, and $\mathrm{pH}$ hemostasis. None of the liver grafts were lost to technical malfunction during the preservation time.

After completion of the first set of 20 human liver transplantations, the results were retrospectively compared to a matched control group of liver transplantations with conventional CS. Patients receiving a perfused graft had decreased AST levels while liver function and survival data were similar in both groups. The authors concluded that normothermic ex vivo liver perfusion using the metra ${ }^{\mathrm{TM}}$ device is safe and logistically feasible for transportation. However, clinical benefit and efficiency have yet to be proven. Therefore, an international multicenter trial has been initiated recently as the next clinical phase.

Another study at the University Medical Centre Groningen, The Netherlands, investigated the feasibility of ex vivo normothermic liver perfusion using a pressure-controlled pulsatile flow [29]. Altogether, four human DCD grafts that had been declined for transplantation were perfused for $6 \mathrm{~h}$. This group allowed an extended cold ischemia time in histidinetryptophan-ketoglutarate (HTK) solution before perfusion (up to $9.5 \mathrm{~h}$ ). The perfusate was composed of packed RBCs and fresh frozen plasma with several nutrient additives. Of note, this group did not use a vasodilator agent in their perfusion. The authors were able to maintain steady vascular flows, with moderate hepatic injury (expressed by perfusate ALT levels and histology). All grafts demonstrated metabolic activity during ex vivo perfusion. Since reperfusion of the grafts was not performed in the study, the clinical benefit of the model needs further evaluation.

More recently, the first model of subnormothermic ex vivo liver perfusion using declined human grafts was introduced by Bruinsma et al. [39]. Three hours of acellular perfusion was performed at $20{ }^{\circ} \mathrm{C}$ in seven DCD liver grafts. The authors observed an increase of energy stores during perfusion even after prolonged CS $(8-15 \mathrm{~h})$. The liver grafts became metabolically active during the first hour of rewarming, demonstrated by an increase in oxygen uptake, lactate hemostasis, and ATP recovery, as well as urea and albumin secretion. At the same time, injury markers increased only moderately. Besides these first experiments in human grafts, this promising technique was only evaluated in a rodent liver reperfusion model [40] and a clinical benefit after transplantation remains unclear.

\section{Normothermic In Situ Recirculation}

Similar to normothermic ex vivo liver perfusion, the main purpose of normothermic in situ recirculation after cardiac arrest is to reverse negative effects of warm ischemic injury. Different to the ex vivo perfusion model is the temporal link to the graft procurement. Usually, one each of an easily accessible major artery and vein (such as iliac artery and vein) is cannulated and blood is recirculated by an extracorporeal membrane oxygenator (ECMO). Except for heparin and the priming fluid of the ECMO, no supplementary additives are needed. Organs are then procured with in situ perfusion similar to heart beating organ retrieval [41]. The fast initiation of the perfusion process allows further graft quality assessment and donor preparation before the actual organ procurement [42]. Recently, some transplant regions have started to transfer donors to a transplant center instead of transporting the organ alone. Thus, transportation of a DCD donor under ECMO recirculation could be possible [43]. The combination of both organ procurement under in situ perfusion and normothermic ex vivo preservation after procurement demonstrated an even more favorable effect on preservation/reperfusion injury and functional transplantation outcomes in grafts exposed to 90 min of warm ischemia [44].

\section{Normothermic In Situ Reperfusion in Clinical Settings}

ECMO support of potential organ donors has been evaluated for three different scenarios: patients declared dead during ECMO therapy, recirculation after controlled cardiac arrest (Maastricht III and IV), or uncontrolled cardiac arrest (Maastricht II, [45]). Therapeutically intended ECMO therapy before declaration of death has not been considered a contraindication for organ donation. It has shown comparable results to conventional graft procurement under heart beating conditions [46, 47]. Initiating ECMO recirculation directly after controlled cardiac arrest in an intensive care unit setting has been described by Magliocca et al. from the University of Michigan [48]. Unfortunately, no outcome data are available for hepatic function and patient survival after transplantation of livers that were procured in this manner.

The strongest data describing the use of in situ recirculation in a DCD setting comes from two Spanish centers [49, 50••]. Patients presenting to the transplant centers with cardiac arrest under mechanical resuscitation were declared dead after $5 \mathrm{~min}$ of hands-off time. In situ recirculation was then initiated using the femoral artery and vein by transcutaneous puncture. After the in situ recirculation was started and oxygenated, organ perfusion was established, suitability for organ donation was evaluated, and permission for organ donation was obtained. After organ procurement, liver grafts were stored in cold University of Wisconsin or Celsior ${ }^{\circledR}$ solution. During the recirculation period, the majority of potential donors were found 
unsuitable after assessment and recirculation was discontinued (91\% of all evaluated patients). One-year graft survival was $70-80 \%$ for organs that would have otherwise never been considered for transplantation. Ischemic-type biliary strictures after transplantation occurred in 5-12\% of the cases.

\section{Limitations}

Despite the promising first results of NMP in liver graft preservation, several limitations of the method have to be considered before a broader application should be considered. First, any perfusion device has to go through a careful safety evaluation. This includes not only its technical liability but also its potential benefit over current preservation techniques. A prospective comparison between the perfusion technique and conventional CS needs to be performed as an intended-to-treat analysis. It is important to determine the number of grafts lost during perfusion due to technical problems. NMP of heart grafts presents a valuable example for the experience of transportable perfusion machines [26]. However, adverse events during preservation are discussed only superficially in scientific publications. Technical incidents, such as cannula perforation or organ misplacements during transportation, came to public attention only by lay newspaper articles [51]. In 2012, the University Clinic Freiburg, Germany, had to suspend their participation in the PROTECT (PROspective multi-center European Trial to Evaluate the safety and performance of the Organ Care System for Heart Transplants) trial for the clinical application of the Organ Care System ${ }^{\mathrm{TM}}$ for several months because a heart graft was lost during preservation [52]. Even in well-established techniques, such as cardiopulmonary bypass machines for cardiac surgery, technical adverse events are rather common (reports accessible through the US FDA homepage [53]). Therefore, technical and logistical difficulties throughout clinical trials of liver perfusion should be expected.

Another risk factor of NMP when compared to CS is the increased chance of bacterial or fungal contamination during the preservation period. Similarly, the use of packed RBCs as oxygen carriers during perfusion bears the risk of virus transmission to the liver graft. Although no reports are available on these matters so far, future clinical trials should examine them.

After technical and clinical benefits have been demonstrated, a cost-efficiency analysis will be necessary for different organ donation scenarios. Since machine perfusion is more complex, it is also far more expensive than CS alone. Not all grafts will benefit from machine preservation. These cases have to be identified to avoid overwhelming costs for the healthcare system.

\section{Future Trends}

Perfusion markers such as flow characteristics, bile production, or transaminase levels have been shown to correlate with post-transplantation outcomes in porcine models [22]. However, further research is necessary to develop reliable criteria to accept or decline grafts based on ex vivo perfusion characteristics. Metabolomic profiling of hepatic biomarkers by mass spectrometry obtained from tissue and perfusate samples presents a promising technique [54-56]. In addition, quantification of hepatic microcirculation [9] and evaluation of tissue oxygenation [57] offer valuable real-time information about the graft condition.

Normothermic ex vivo perfusion creates an optimal environment where protective strategies against preservation/ reperfusion injury can be applied prior to organ transplantation [58]. Protective strategies include the delivery of simple radical scavenging molecules such as $\mathrm{N}$-acetylcysteine or tocopherol; the introduction of protective enzymes such as hemoxigenase-1 [59]; and the use of complex immunomodulation therapy such as vector gene delivery [60], antisense nucleotides [61, 62], or stem-cell therapy [63, 64]. In addition, defatting strategies have been described for normothermic perfusion of steatotic grafts in rats and pigs [ 65 , 66]. The options for graft pretreatment are not limited to preservation injury. It could also be extended to immunomodulation to reduce rejection after transplantation or to prevent disease recurrence such as hepatitis $\mathrm{C}$ virus or hepatocellular carcinoma.

Further modifications of the perfusate are also likely. One foreseeable trend is the use of an artificial oxygen carrier as a substitution for packed RBCs [67]. Thereby, the infectious risk factor could be reduced and all components of the perfusate would be easily accessible and storable.

\section{Conclusions}

Over the last two decades, the organ shortage has resulted in an increased use of marginal grafts in order to meet the overwhelming demand for donor organs. The standard preservation method of CS is associated with severe graft injury and poor graft function in marginal grafts, indicating the limitations of this conventional preservation technique. The problems associated with CS are a major obstacle to increasing the donor pool and to reducing waiting list mortality. Normothermic liver perfusion is a novel alternative preservation technique for marginal grafts. It offers the opportunity to decrease cold ischemic injury and to assess the organ quality and function of a marginal graft prior to transplantation. In addition, normothermic perfused liver grafts are accessible for repair strategies, opening up a new field of pre-transplant organ conditioning. Initial clinical trials demonstrated that both normothermic ex vivo perfusion of marginal grafts as well as normothermic in situ blood circulation before organ retrieval after cardio-circulatory death are feasible methods 
showing promising preliminary results. A combination of both methods could further improve organ preservation in the future. Normothermic liver perfusion is still in an early stage of clinical development and its superiority over existing preservation methods has yet to be proven. Possible failures of perfusion machines during transportation, the overall cost efficiency, and the clinical benefit need further evaluation.

Larger clinical trials are currently in progress to determine the efficacy and limitations of this new preservation method. Normothermic ex vivo perfusion offers new opportunities for liver transplantation and has the potential to change organ preservation within the next decade.

\section{Compliance with Ethics Guidelines}

Conflict of Interest Vinzent N. Spetzler, Nicolas Goldaracena, Nazia Selzner, and Markus Selzner declare that they have no conflict of interest.

Human and Animal Rights and Informed Consent This article does not contain any studies with human or animal subjects performed by any of the authors.

\section{References}

Papers of particular interest and published recently are highlighted below as:

- Of importance

•- Of major importance

1. Collins GM, Halasz NA. Letter: Composition of intracellular flush solutions for hypothermic kidney storage. Lancet. 1975;1(7900): 220.

2. Starzl TE, Groth CG, Brettschneider L, et al. Orthotopic homotransplantation of the human liver. Ann Surg. 1968;168(3):392415.

3. Vajdova K, Smrekova R, Mislanova C, et al. Cold-preservationinduced sensitivity of rat hepatocyte function to rewarming injury and its prevention by short-term reperfusion. Hepatology. 2000;32(2):289-96.

4. Benkoel L, Dodero F, Hardwigsen J, et al. Effect of ischemiareperfusion on $\mathrm{Na}^{+}, \mathrm{K}+$-ATPase expression in human liver tissue allograft: image analysis by confocal laser scanning microscopy. Dig Dis Sci. 2004;49(9):1387-93.

5. Lee SM, Park MJ, Cho TS, et al. Hepatic injury and lipid peroxidation during ischemia and reperfusion. Shock. 2000;13(4):27984.

6. Ilhan N, Halifeoglu I, Ozercan HI, et al. Tissue malondialdehyde and adenosine triphosphatase level after experimental liver ischaemia-reperfusion damage. Cell Biochem Funct. 2001;19(3): 207-12.

7. Datta G, Fuller BJ, Davidson BR. Molecular mechanisms of liver ischemia reperfusion injury: insights from transgenic knockout models. World J Gastroenterol. 2013;19(11):1683-98.

8. van Golen RF, van Gulik TM, Heger M. Mechanistic overview of reactive species-induced degradation of the endothelial glycocalyx during hepatic ischemia/reperfusion injury. Free Radic Biol Med. 2012;52(8):1382-402.
9. Vollmar B, Menger MD. The hepatic microcirculation: mechanistic contributions and therapeutic targets in liver injury and repair. Physiol Rev. 2009;89(4):1269-339.

10. Elias-Miro M, Jimenez-Castro MB, Rodes J, et al. Current knowledge on oxidative stress in hepatic ischemia/reperfusion. Free Radic Res. 2013;47(8):555-68.

11. Fondevila C, Busuttil RW, Kupiec-Weglinski JW. Hepatic ischemia/reperfusion injury-a fresh look. Exp Mol Pathol. 2003;74(2):86-93.

12. Cursio R, Gugenheim J. Ischemia-reperfusion injury and ischemictype biliary lesions following liver transplantation. J Transplant. 2012;2012:164329.

13. Seehofer D, Eurich D, Veltzke-Schlieker W, et al. Biliary complications after liver transplantation: old problems and new challenges. Am J Transplant. 2013;13(2):253-65.

14. Rhee J, Kern B, Cooper J, et al. Organ donation. Semin Liver Dis. 2009;29(1):19-39.

15. Pratschke S, Loehe F, Graeb C, et al. Usage of marginal organs for liver transplantation: a way around the critical organ shortage? [in German]. Zentralbl Chir. 2009;134(2):107-12.

16. Chen XB, Xu MQ. Primary graft dysfunction after liver transplantation. Hepatobiliary Pancreat Dis Int. 2014;13(2):125-37.

17. Feng S, Goodrich NP, Bragg-Gresham JL, et al. Characteristics associated with liver graft failure: the concept of a donor risk index. Am J Transplant. 2006;6(4):783-90.

18. Vogel T, Brockmann JG, Coussios C, et al. The role of normothermic extracorporeal perfusion in minimizing ischemia reperfusion injury. Transplant Rev (Orlando). 2012;26(2):156-62.

19. Boehnert MU, Yeung JC, Bazerbachi F, et al. Normothermic acellular ex vivo liver perfusion reduces liver and bile duct injury of pig livers retrieved after cardiac death. Am J Transplant. 2013;13(6): 1441-9. This paper provides the first histological proof of biliary protection through normothermic ex vivo liver perfusion.

20. Reddy SP, Bhattacharjya S, Maniakin N, et al. Preservation of porcine non-heart-beating donor livers by sequential cold storage and warm perfusion. Transplantation. 2004;77(9):1328-32.

21. Schön MR, Kollmar O, Wolf S, et al. Liver transplantation after organ preservation with normothermic extracorporeal perfusion. Ann Surg. 2001;233(1):114-23.

22. Brockmann J, Reddy S, Coussios C, et al. Normothermic perfusion: a new paradigm for organ preservation. Ann Surg. 2009;250(1):16.

23. Balfoussia D, Yerrakalva D, Hamaoui K, et al. Advances in machine perfusion graft viability assessment in kidney, liver, pancreas, lung, and heart transplant. Exp Clin Transplant. 2012;10(2):87100.

24. Machuca TN, Cypel M, Keshavjee S. Advances in lung preservation. Surg Clin North Am. 2013;93(6):1373-94.

25. Warnecke G, Moradiellos J, Tudorache I, et al. Normothermic perfusion of donor lungs for preservation and assessment with the Organ Care System lung before bilateral transplantation: a pilot study of 12 patients. Lancet. 2012;380(9856):1851-8.

26. Messer S, Ardehali A, Tsui S. Normothermic donor heart perfusion: current clinical experience and the future. Transpl Int. 2014. doi:10. 1111/tri. 12361.

27. Nicholson ML, Hosgood SA. Renal transplantation after ex vivo normothermic perfusion: the first clinical study. Am J Transplant. 2013;13(5):1246-52.

28. Porepa LF, Starling RC. Destination therapy with left ventricular assist devices: for whom and when? Can J Cardiol. 2014;30(3): 296-303.

29. Op den Dries S, Karimian N, Porte RJ. Normothermic machine perfusion of discarded liver grafts. Am J Transplant. 2013;13(9): 2504. 
30. Butler AJ, Rees MA, Wight DG, et al. Successful extracorporeal porcine liver perfusion for $72 \mathrm{hr}$. Transplantation. 2002;73(8): 1212-8.

31. Knaak JM, Spetzler VN, Goldaracena N, et al. Subnormothermic ex vivo liver perfusion reduces endothelial cell and bile duct injury after donation after cardiac death pig liver transplantation. Liver Transpl. 2014;20(11):1296-305.

32. Knaak JM, Spetzler VN, Goldaracena N, et al. Technique of subnormothermic ex vivo liver perfusion for the storage, assessment, and repair of marginal liver grafts. J Vis Exp. 2014;90: e51419.

33. Bellomo R, Suzuki S, Marino B, et al. Normothermic extracorporeal perfusion of isolated porcine liver after warm ischaemia: a preliminary report. Crit Care Resusc. 2012;14(3):173-6.

34. Nasser A, Liu Q, Farias K, et al. The effect of normothermic machine perfusion (NMP) using various perfusates on the hepatocellular and billiary epithelial integrity in porcine donation after cardiac death (DCD) models. Liver Transpl. 2013;19 Suppl 1:S108.

35. Friend PJ, Imber C, St Peter S, et al. Normothermic perfusion of the isolated liver. Transplant Proc. 2001;33(7-8):3436-8.

36. Gravante G, Ong SL, Metcalfe MS, et al. Cytokine response to ischemia/reperfusion injury in an ex vivo perfused porcine liver model. Transplant Proc. 2009;41(4):1107-12.

37. Nassar A, Liu Q, Farias K, et al. Role of vasodilation during normothermic machine perfusion of DCD porcine livers. Int $\mathrm{J}$ Artif Organs. 2014;37(2):165-72.

38.• Ravikumar R, Jassem W, Mergental H, et al.: Liver transplantation after ex-vivo normothermic liver perfusion. At: Joint International Congress of ILTS, ELITA and LICAGE; 4-7 Jun 2014; London, UK. This group recently presented the first clinical results of ex vivo liver perfusion at the International Liver Transplantation Society (ILTS) meeting in London. The first full journal publication is still waited for.

39. Bruinsma BG, Yeh H, Ozer S, et al. Subnormothermic machine perfusion for ex vivo preservation and recovery of the human liver for transplantation. Am J Transplant. 2014;14(6):1400-9.

40. Bruinsma BG, Berendsen TA, Izamis ML, et al. Determination and extension of the limits to static cold storage using subnormothermic machine perfusion. Int J Artif Organs. 2013;36(11):775-80.

41. Garcia-Valdecasas JC, Fondevila C. In-vivo normothermic recirculation: an update. Curr Opin Organ Transplant. 2010;15(2):173-6.

42. Valero R, Garcia-Valdecasas JC, Tabet J, et al. Hepatic blood flow and oxygen extraction ratio during normothermic recirculation and total body cooling as viability predictors in non-heart-beating donor pigs. Transplantation. 1998;66(2):170-6.

43. Doyle MB, Vachharajani N, Wellen JR, et al. A novel organ donor facility: a decade of experience with liver donors. Am J Transplant. 2014;14(3):615-20.

44. Fondevila C, Hessheimer AJ, Maathuis MH, et al. Superior preservation of DCD livers with continuous normothermic perfusion. Ann Surg. 2011;254(6):1000-7.

45. Daemen JW, Kootstra G, Wijnen RM, et al. Nonheart-beating donors: the Maastricht experience. Clin Transpl. 1994;11(12): 303-16.

46. Mourad MM, Reay M, Muiesan P, et al. Patient with liver dysfunction while maintained on veno-venous extracorporeal membrane oxygenation should not be overlooked as a potential donor. Transpl Int. 2014;27(6):e50-3.

47. Bodzin AS, Hirose $\mathrm{H}$, West $\mathrm{S}$, et al. Outcome of organs procured from donors on extracorporeal membrane oxygenation support: an analysis of kidney and liver allograft data. Clin Transplant. 2014;28(7):816-20
48. Magliocca JF, Magee JC, Rowe SA, et al. Extracorporeal support for organ donation after cardiac death effectively expands the donor pool. J Trauma. 2005;58(6):1095-101. discussion 101-2.

49. Jiménez-Galanes S, Meneu-Diaz MJ, Elola-Olaso AM, et al. Liver transplantation using uncontrolled non-heart-beating donors under normothermic extracorporeal membrane oxygenation. Liver Transpl. 2009;15(9):1110-8.

50.• Fondevila C, Hessheimer AJ, Flores E, et al. Applicability and results of Maastricht type 2 donation after cardiac death liver transplantation. Am J Transplant. 2012;12(1):162-70. This group took a leading role in the development of normothermic in situ perfusion; this paper provides the results of almost 8 years of clinical experience in Maastricht II DCD donation.

51. Haarhoff H. Damit das Herz frisch bleibt [to keep the heart fresh]. TAZ. 2012;9926:5.

52. Bundesärztekammer. Einsatz eines Perfusionssystems für Spenderherzen [use of a perfusion system for heart grafts]. Tätigkeitsbericht 2012 der Bundesärztekammer. Hannover: Bundesärzekammer; 2013. p. 299.

53. U.S. Food and Drug Administration. MAUDE - Manufacturer and User Facility Device Experience. 2014. http://www.accessdata.fda. gov/scripts/cdrh/cfdocs/cfmaude/textsearch.cfm. Accessed Jul 2014.

54. Cortes M, Pareja E, Garcia-Canaveras JC, et al. Metabolomics discloses donor liver biomarkers associated with early allograft dysfunction. J Hepatol. 2014;61(3):564-74.

55. Bojko B, Gorynski K, Gomez-Rios GA, et al. Low invasive in vivo tissue sampling for monitoring biomarkers and drugs during surgery. Lab Investig. 2014;94(5):586-94.

56. Bojko B, Gorynski K, Gomez-Rios GA, et al. Solid phase microextraction fills the gap in tissue sampling protocols. Anal Chim Acta. 2013;803:75-81.

57. Cyrous A, O'Neal B, Freeman WD. New approaches to bedside monitoring in stroke. Expert Rev Neurother. 2012;12(8):915-28.

58. Zhai Y, Petrowsky H, Hong JC, et al. Ischaemia-reperfusion injury in liver transplantation-from bench to bedside. Nat Rev Gastroenterol Hepatol. 2013;10(2):79-89.

59. Sass G, Barikbin R, Tiegs G. The multiple functions of heme oxygenase-1 in the liver. Z Gastroenterol. 2012;50(1):34-40.

60. Cypel M, Liu M, Rubacha M, et al. Functional repair of human donor lungs by IL-10 gene therapy. Sci Transl Med. 2009;1(4):4ra9.

61. Ponnappa BC, Israel Y, Aini M, et al. Inhibition of tumor necrosis factor alpha secretion and prevention of liver injury in ethanol-fed rats by antisense oligonucleotides. Biochem Pharmacol. 2005;69(4):569-77.

62. Thomas M, Deiters A. MicroRNA miR-122 as a therapeutic target for oligonucleotides and small molecules. Curr Med Chem. 2013;20(29):3629-40.

63. Deng $\mathrm{L}$, Liu G, Wu X, et al. Adipose derived mesenchymal stem cells efficiently rescue carbon tetrachloride-induced acute liver failure in mouse. Sci. World J. 2014;103643: 1-8.

64. Van Raemdonck D, Neyrinck A, Rega F, et al. Machine perfusion in organ transplantation: a tool for ex-vivo graft conditioning with mesenchymal stem cells? Curr Opin Organ Transplant. 2013;18(1):24-33.

65. Jamieson RW, Zilvetti M, Roy D, et al. Hepatic steatosis and normothermic perfusion-preliminary experiments in a porcine model. Transplantation. 2011;92(3):289-95.

66. Liu Q, Berendsen T, Izamis ML, et al. Perfusion defatting at subnormothermic temperatures in steatotic rat livers. Transplant Proc. 2013;45(9):3209-13.

67. Nui A, Katsuramaki T, Kikuchi $\mathrm{H}$, et al. The functional integrity of a normothermic perfusion system using artificial blood in pig liver. J Surg Res. 2006;131(2):189-98. 